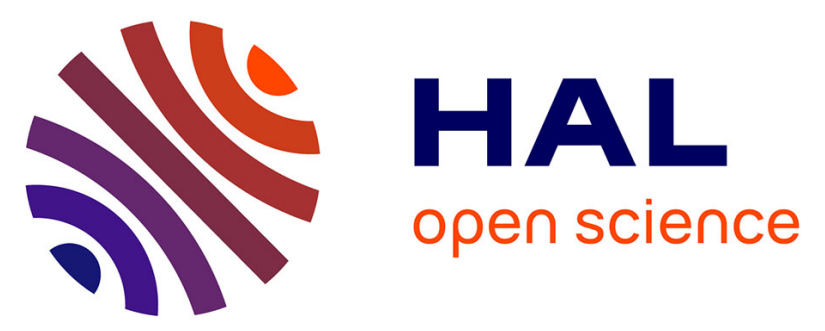

\title{
Fast and stable photoacoustic gas spectroscopy by frequency tracking of a quartz tuning fork in closed loop detection
}

Maxime Duquesnoy, Raphaël Lévy, Jean-Michel Melkonian, Guillaume Aoust, Myriam Raybaut, Antoine Godard

\section{To cite this version:}

Maxime Duquesnoy, Raphaël Lévy, Jean-Michel Melkonian, Guillaume Aoust, Myriam Raybaut, et al.. Fast and stable photoacoustic gas spectroscopy by frequency tracking of a quartz tuning fork in closed loop detection. Imaging and Applied Optics. 3D Image Acquisition and Display: Technology, Perception and Applications, Jun 2020, Washington, United States. pp.JTu2A.16, 10.1364/3D.2020.JTu2A.16 . hal-03152477

\section{HAL Id: hal-03152477 \\ https://hal.science/hal-03152477}

Submitted on 25 Feb 2021

HAL is a multi-disciplinary open access archive for the deposit and dissemination of scientific research documents, whether they are published or not. The documents may come from teaching and research institutions in France or abroad, or from public or private research centers.
L'archive ouverte pluridisciplinaire HAL, est destinée au dépôt et à la diffusion de documents scientifiques de niveau recherche, publiés ou non, émanant des établissements d'enseignement et de recherche français ou étrangers, des laboratoires publics ou privés. 


\title{
Fast and stable photoacoustic gas spectroscopy by frequency tracking of a quartz tuning fork in closed loop detection
}

\author{
Maxime Duquesnoy ${ }^{1,2}$, Raphaël Lévy ${ }^{2}$, Jean-Michel Melkonian ${ }^{2}$, \\ Guillaume Aoust ${ }^{1}$, Myriam Raybaut ${ }^{2}$, Antoine Godard ${ }^{2}$ \\ ${ }^{1}$ Mirsense, Nanno-INNOV, building 863, 8 Avenue de la Vauve, 91120 Palaiseau, France \\ ${ }^{2}$ DPHY, ONERA, Université Paris Saclay, F-91123 Palaiseau, France \\ Author e-mail address: maxime.duquesnoy@mirsense.com
}

\begin{abstract}
We previously introduced a new QEPAS (Quartz Enhanced Photoacoustic Spectroscopy) signal processing allowing fast and stable measurements. We now present improvements of our measurement method on sensitivity and measurement time. (C) 2020 The Authors
\end{abstract}

\section{Motivation}

Trace gas detection can find application in assessment of air quality, detection of pollutants or hazardous gases, control of exhaust gases, monitoring of industrial processes, etc. However, the available gas sensors on the market are either, very sensitive but bulky and expensive, or very compact but with a lack of sensitivity or selectivity. This is also true for devices using laser detection, even though they bring advantages in terms of accuracy, selectivity, speed, and low drift measurements.

Photoacoustic spectroscopy is a technique that could create a compromise between size, sensitivity and cost and allows a simultaneous detection of multiple species. It consists in the excitation of molecules by a modulated laser source, creating a periodical heating of the medium. This heating induces acoustic waves that can be detected by a mechanical transducer, such as a microphone membrane, a cantilever or a tuning fork.

Quartz Enhanced Photoacoustic Spectroscopy (QEPAS) is a particular implementation of photoacoustic spectroscopy in which a quartz tuning fork converts the acoustic waves into a piezoelectric signal. QEPAS is a recent technique which appeared in 2002 [1]. Compared to the other photoacoustic techniques, QEPAS exhibits a better isolation to acoustic and vibrational background. Nonetheless, the use of high quality factor tuning forks $(\mathrm{Q}>$ 8000 in air) makes the measurements slow (a few seconds), and the sensor even more sensitive to temperature induced-drifts leading to measurement biases or even signal dropout. In order to circumvent these problems new detection techniques arose such as Beat Frequency QEPAS [2] or frequency tracking of the tuning fork [3,4].

We already presented a new detection principle [5] allowing to increase the measurement rate and stability. This method relies on the use of frequency counting instead of classical lock-in detection. In this paper we will present a further study of our detection method taking into account improvements we have identified.

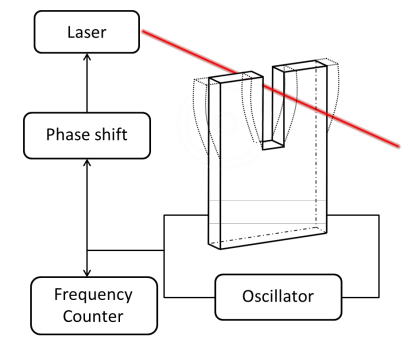

Fig. 1. Schematic of the method principle.

\section{Principle of operation}

Our measurement method relies on the fact that a force applied to a self-sustained resonator induces a frequency shift which is proportional to that force.

The tuning fork vibration is maintained at resonance by means of an electrical oscillator (see Fig.1). When the laser is tuned to a gas absorption line, a photoacoustic force is created and applied to the inner surfaces of the beams of the tuning fork with the appropriate phase compared to the oscillator generated force, inducing a frequency shift 
in the oscillator loop. The technique is linear so that the frequency shift is directly proportional to the gas concentration. This technique also allows differential measurements for the correction of drifts and cancellation of background noises.

\section{First results and future improvements}
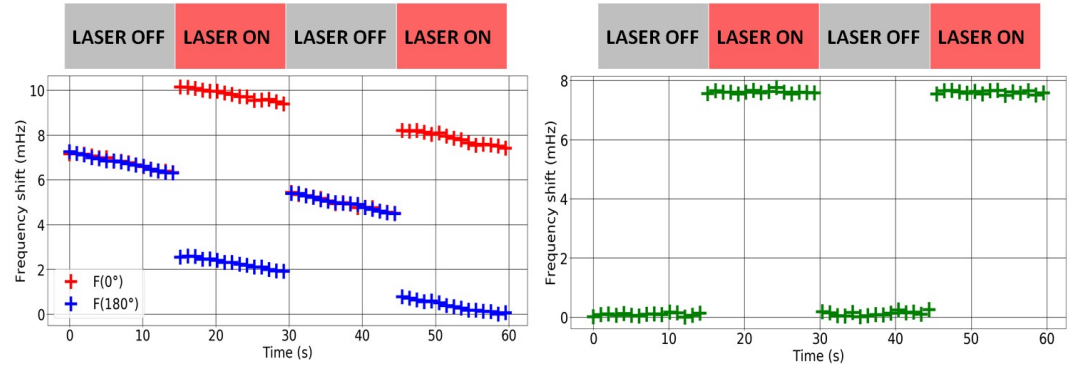

Fig. 2. First measurements made with our method. Measurement of frequency shifts for two different phases, with a frequency drift (left). Differential frequency shift with cancellation of the drift over time (right).

A proof of principle was presented in [5] (see Fig.2). By exciting the resonator with a custom analogical electrical loop we demonstrated that the method enables differential measurements while tracking the tuning fork resonance frequency, consequently improving the sensor stability over time. In addition, placing the tuning fork at resonance allows making measurements faster, passing from several seconds for classic detection down to 50 $\mathrm{ms}$ for our method. However, the sensitivity of the system was NNEA $=3.7 \times 10^{-7} \mathrm{~W} \cdot \mathrm{cm}^{-1} \cdot \mathrm{Hz}^{-1 / 2}$ which is one order of magnitude worse than the classical method with the same tuning fork [6]. In order to improve our detection method, we identified several factors that could lead to an improvement of sensitivity or speed of measurement as well as accuracy. The electrical excitation force of the resonator has to be reduced, what we achieved by modifying the tuning fork electrode pattern and using a numerical electrical oscillator loop based on FPGA (Field Programmable Gate Arrays). Indeed, the excitation electrode surface was minimized while keeping a sufficient excitation force (see Fig.3). By doing so, we increased the excitation electrode resistance by a factor 5 .

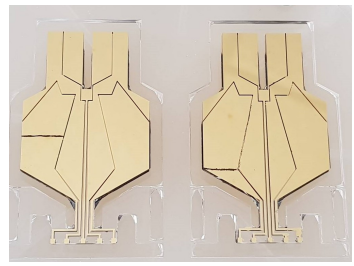

Fig. 3. Modifications of electrode pattern on two custom tuning forks.

Also, passing from an analogical oscillator loop to a numerical one allows reducing consequently the excitation voltage of the tuning fork which we hope will permit to equal the sensitivity of the classical detection method. By using a commercial lock-in detection HF2LI (Zurich Instruments), we propose to analyze the ultimate sensitivity of our sensor on $\mathrm{C}_{2} \mathrm{H}_{2}$ in different configurations as well as the possibility to reduce measurement time.

\section{References}

[1] A. A. Kosterev, Y. A. Bakhirkin, R. F. Curl, and F. K. Tittel, “Quartz-enhanced photoacoustic spectroscopy,” Opt. Lett., vol. 27, no. 21, p. 1902,2002

[2] H. Wu, L. Dong, X. Yin, H. Zheng, S. Jia, and F. K. Tittel, "Fast and calibration-free trace-gas monitoring based on beat frequency quartz-enhanced photoacoustic spectroscopy,” vol. 2018, no. Dc, pp. 5-6, 2018.

[3] R. Rousseau, N. Maurin, W. Trzpil, M. Bahriz, and A. Vicet, "Quartz Tuning Fork Resonance Tracking and application in Quartz Enhanced Photoacoustics Spectroscopy," pp. 1-11, 2019.

[4] M. Mordmueller, W. Schade, and U. Willer, "QEPAS with electrical co-excitation for photoacoustic measurements in fluctuating background gases," Appl. Phys. B Lasers Opt., vol. 123, no. 8, pp. 1-9, 2017.

[5] R. Levy, M. Duquesnoy, G. Aoust, J. M. Melkonian, and M. Raybaut, "New signal processing for fast and precise QEPAS measurements," IEEE Trans. Ultrason. Ferroelectr. Freq. Control, vol. PP, no. c, pp. 1-1, 2019.

[6] M. Duquesnoy, G. Aoust, J. M. Melkonian, R. Lévy, M. Raybaut, and A. Godard, "Quartz enhanced photoacoustic spectroscopy based on a custom quartz tuning fork," Sensors (Switzerland), vol. 19, no. 6, pp. 1-10, 2019. 